\title{
Operative Intervention for a Chronic Fifth Carpometacarpal Dislocation: A Case Report and Review of Literature
}

\author{
Andrew D Boltuch ${ }^{1}$, John N Harker ${ }^{1,2}$
}

Learning Point of the Article:

The identification and treatment of chronic fifth carpometacarpal dislocations.

\section{Abstract}

Introduction: Carpometacarpal (CMC) dislocations are infrequent hand injuries that are often missed in the acute care setting, resulting in inappropriate treatment and significant morbidity. CMC dislocations occur in a volar or dorsal direction, and volar dislocations are traditionally subclassified as either volar-ulnar or volar-radial. While various treatment methods and injury identification techniques have been reported in the literature, there remains a lack of standardization in the treatment of these injuries. This case report reviews the current literature on the subject in an attempt to further our knowledge on the subject.

Case Report: A 73-year-old right hand dominant male presented to the clinic complaining of persistent pain on the ulnar aspect of his left hand for the past 3 months. The injury had been missed in the urgent care and emergency settings before his arrival to the office. Radiographs demonstrated a volar-ulnar dislocation of the fifth CMC joint. The patient subsequently underwent open reduction with internal fixation to correct his deformity. Post-operative QuickDASH and grip strength measurements were gathered at post-operative visits.

Conclusion: This paper provides a literature review on the diagnostic techniques and management options of the fifth CMC dislocations. It further provides a treatment algorithm for a chronic volar-ulnar fifth CMC dislocation being amenable to open reduction and Kirschner wire fixation with an excellent outcome.

Keywords: Carpometacarpal, hand, dislocation.

\section{Introduction}

Isolated carpometacarpal (CMC) dislocations are rare injuries and are frequently missed in the emergency department and urgent care settings. Current literature on this specific injury varies with regard to identification techniques, treatment methodology, and post-treatment outcomes. This paper will present a rare case of an isolated, chronic, volar-ulnar fifth CMC dislocation treated with open reduction and Kirschner wire fixation. There will then be a review of literature following the case report focusing on diagnostic techniques, classification systems, treatment methods, and clinical outcomes.

\section{Case Report}

A 73-year-old right hand dominant male presented to the clinic complaining of persistent pain on the ulnar aspect of his left hand. His symptoms began 3 months prior, after falling onto the ulnar aspect of his hand. The patient had been evaluated at the time of injury in the emergency department and subsequently at an urgent care facility where the correct diagnosis remained unidentified despite obtaining the radiographic images shown in (Fig. 1a, 1b).

3 months after his initial injury, the patient presented to the senior author's office with the primary complaint of persistent pain at the base of his fifth metacarpal. He also noted that his small finger would get caught outside his pocket when retrieving an item. On physical examination of the left hand, a slight small finger abduction deformity was noted throughout digital range of motion. There was tenderness to palpation over

\begin{tabular}{|c|}
\hline Access this article online \\
\hline $\begin{array}{c}\text { Website: } \\
\text { www.jocr.co.in }\end{array}$ \\
\hline DOI: \\
$10.13107 /$ jocr.2021.v11.01.1986 \\
\hline
\end{tabular}

Department of Orthopaedic Surgery, Largo Medical Center, Largo, Florida, United States of America,

'Department of Orthopaedic, Clearwater Orthopedic and Sports Medicine, Clearwater, Florida, United States of America.

Address of Correspondence:

Dr. Andrew D Boltuch,

Doctor of Osteopathic Medicine, Department of Orthopaedic Surgery, Largo Medical Center 201 14th Street SW, Largo, Florida - 33770, United States of America.

E-mail: andrew.boltuch@gmail.com

Journal of Orthopaedic Case Reports | pISSN 2250-0685 | eISSN 2321-3817 | Available on www.jocr.co.in | doi:10.13107/jocr.2021.v11.i01.1986 This is an Open Access article distributed under the terms of the Creative Commons Attribution Non-Commercial License (http://creativecommons.org/licenses/by-nc/3.0) which permits unrestricted non-commercial use, distribution, and reproduction in any medium, provided the original work is properly cited. 


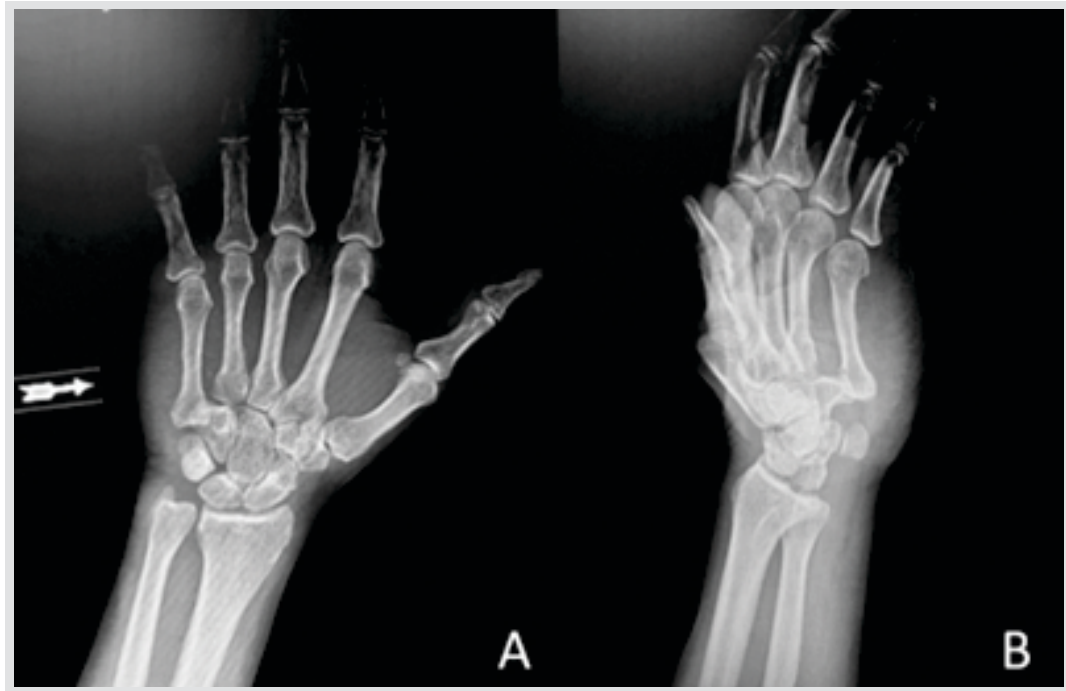

Figure 1: Injury radiographs. (a) Posteroanterior radiograph of the left hand demonstrating an ulnar and proximal deviation at the base of the fifth metacarpal with overlap of the hamate articular surface taken at the time of injury; (b) Oblique radiograph of the left hand demonstrating volar deviation at the base of the fifth metacarpal.

the base of the metacarpal and no signs of neurological or vascular impairment. Posteroanterior (PA), lateral, and oblique radiographs reviewed from the time of injury and those taken at the time of presentation demonstrated a dislocation of the fifth metacarpal base in a volar and ulnar direction.

The decision was made with the patient to proceed with operative reduction and fixation of his chronic injury. A longitudinal incision was made along the dorsal aspect of the fifth metacarpal. The extensor tendons were protected and retracted. Surrounding intrinsic musculature and fibrous tissue were released from the metacarpal allowing visualization of the CMC joint. Successful reduction was accomplished with axial traction and manual pressure applied in a palmar to dorsal direction at the base of the metacarpal. An appropriate reduction was fluoroscopically confirmed at the metacarpal base, and the abduction deformity was reduced. The fifth metacarpal was then stabilized with parallel Kirschner wires to the fourth metacarpal distally and proximally. The reduction was confirmed and supported with an additional Kirschner wire driven retrograde through the $\mathrm{CMC}$ joint into the hamate (Fig. 2).

The patient was immobilized in an ulnar gutter splint and pins were maintained for 6 weeks. He was given a home exercise

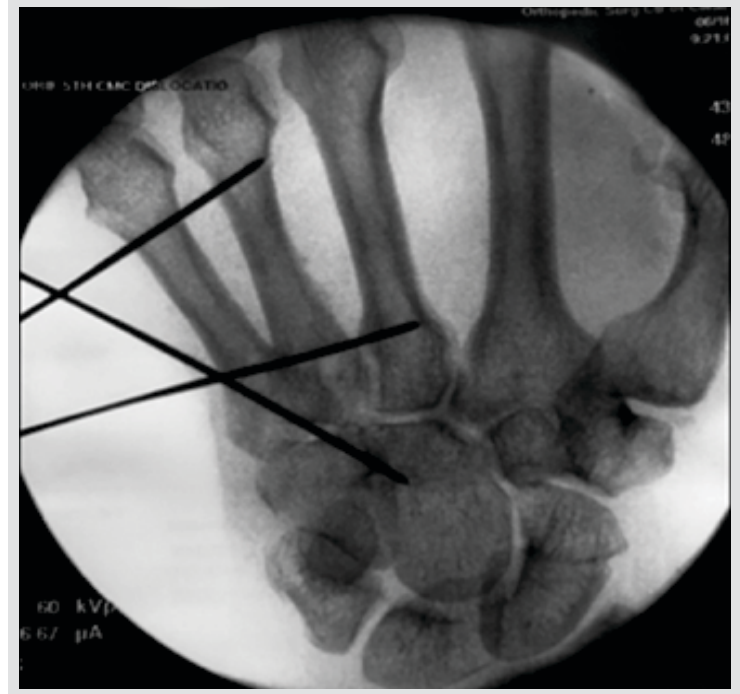

Figure 2: Intraoperative radiographs. Intraoperative posteroanterior radiograph demonstrating Kirschner wire fixation of the left fifth metacarpal following open reduction of the fifth carpometacarpal joint.

program to begin following pin removal. The patient was followed postoperatively at 6 weeks, 10 weeks, and 6 months. Clinical improvement was assessed by measuring QuickDASH scores and grip strengths measured with a Jamar grip dynamometer, as shown in (Table 1). The patient's QuickDASH score was 6.8 at his 10-week follow-up and 4.5 at his 6-month follow-up. At 6 months, the patient's grip strength had returned to normal. He was pain free, achieved full range of motion in his fingers and wrist, and the reduction was well maintained. The patient was very satisfied with his treatment and post-operative outcome. Radiographs and clinical photographs were taken at the patient's 6-month post-operative follow-up, as shown in (Fig. 3,4), respectively.

\section{Discussion}

Isolated dislocations of the fifth metacarpal are rare injuries that require appropriate identification and reduction to restore orientation and function to the hand. The previous literature on the diagnosis of metacarpal dislocations has identified various methods for the identification of this pathology.

Fisher et al. described four radiographic criteria to evaluate for metacarpal dislocations on a PA view. They describe factors

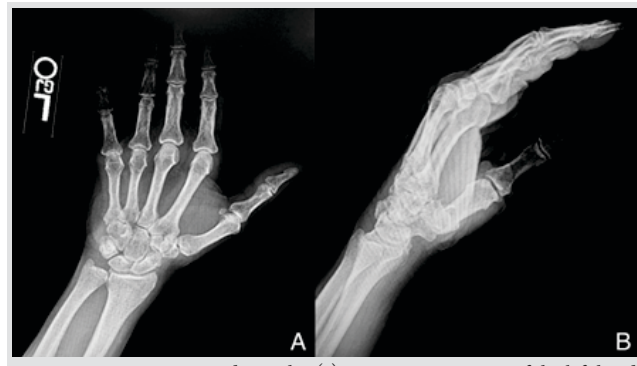

Figure 3: Post-operative radiographs. (a) Posteroanterior view of the left han taken 6 months postoperatively; (b) lateral view of the left hand taken 6 month postoperatively demonstrate maintained reduction of the fifth carpometacarpal joint.
Table 1: Comparison of grip strength between affected and unaffected hands at multiple time points

\begin{tabular}{|c|c|c|c|}
\hline Grip position & 1 & 3 & 5 \\
\hline $\begin{array}{c}\text { Grip strength } \\
10 \text { weeks post-operative }(\mathrm{kg})\end{array}$ & 8 & 14 & 10 \\
\hline 6 months post-operative $(\mathrm{kg})$ & 18 & 32 & 26 \\
\hline Unaffected hand $(\mathrm{kg})$ & 17 & 29 & 19 \\
\hline
\end{tabular}



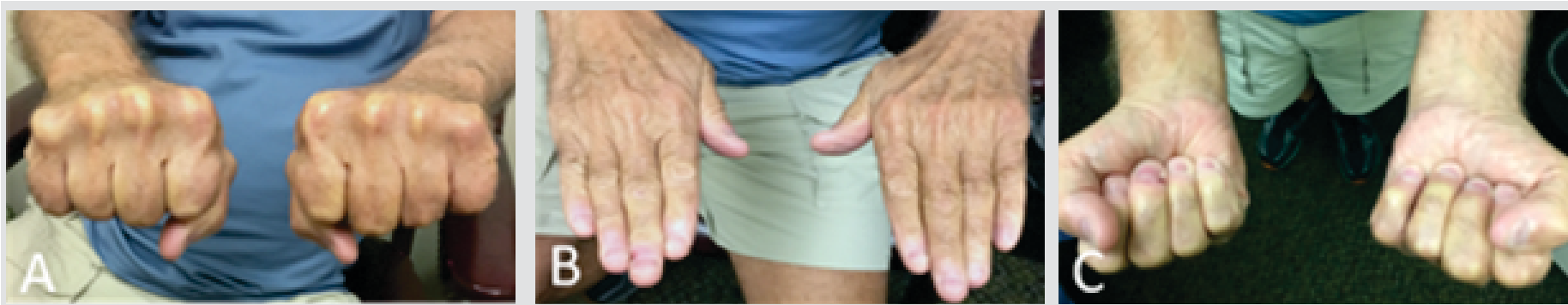

Figure 4: Post-operative clinical photographs. (a) Clenched fist view; (b) dorsal finger extension view; (c) supinated clenched fist view taken 6 months status post left fifth metacarpal open reduction with Kirschner wire fixation. Images demonstrate full range of motion with a minor residual abduction of the small digit noted in the supinated clenched fist view.

such as parallelism of the articular surfaces, symmetric joint spaces at the CMC joints measuring 1-2 mm, no overlap of opposing articular surfaces, and a distinct cortical rim at the $\mathrm{CMC}$ joints. They further describe a Parallel M, which is drawn along the opposing articular surfaces of the CMC joints. When concerned about the lateral metacarpals, a 30 degree pronated lateral radiograph helps bring the fifth CMC joint into direct visualization $[1,2]$. Mcdonald et al. also discussed the intermetacarpal angle, which is measured on a lateral radiograph between the index/small and long/small medullary canals, which, if $>10$ degrees, is suggestive of a metacarpal dislocation [3]. However, this technique has been shown to have decreased sensitivity for dislocations in a volar direction [4]. Finally, because of radiographic difficulty in providing the appropriate diagnosis, Wantable et al. recommended a computed tomography (CT) scan in the workup of a CMC dislocation [5].

Another method to identify this deformity is the metacarpal cascade line, which is drawn down the central longitudinal axis of each metacarpal [6]. These lines should all converge to a common point approximately $2 \mathrm{~cm}$ proximal to the articular surface of the distal radius. Any alteration from this convergence point suggests a metacarpal dislocation. The authors of this paper found that the metacarpal cascade line was an easily reproducible radiographic finding which quickly demonstrated the presence of this metacarpal dislocation (Fig. 5).

Once the appropriate diagnosis is made, it is essential to understand the relevant disrupted anatomy and potential for instability. CMC dislocations of the fifth metacarpal are classified into dorsal or volar dislocations. Nalebuff further subclassified volar dislocations into volar-radial and volar-ulnar dislocations. Volar-radial dislocations are associated with the loss of all ligamentous and tendinous attachments. This dislocation pattern may be more challenging to reduce, and open reduction is usually required, although reports of successful closed reduction have been made $[3,7,8]$. Volarulnar dislocations are associated with loss of the CMC and intermetacarpal ligaments with preservation of the pisometacarpal ligament, the flexor carpi ulnaris, and the extensor carpi ulnaris tendon insertions. This pattern tends to be more easily reduced; however, maintenance of reduction traditionally requires continuous traction or Kirschner wire fixation $[1,9]$. Deforming forces on the metacarpal include proximal pull by the direct insertion of the extensor carpi ulnaris and the indirect pull of the flexor carpi ulnaris through the pisometacarpal ligament. Stability to the fifth CMC joint is primarily conferred by the intermetacarpal ligament. Partial sectioning of this ligament allows joint subluxation, and complete sectioning is required for a volar joint dislocation. Both of these injury patterns include damage to this ligament, creating an environment of instability $[9,8,10]$.

Several studies have shown that acute CMC dislocations can be successfully treated with closed treatment methods $[3,7,8]$. The fracture reduction technique involves applying

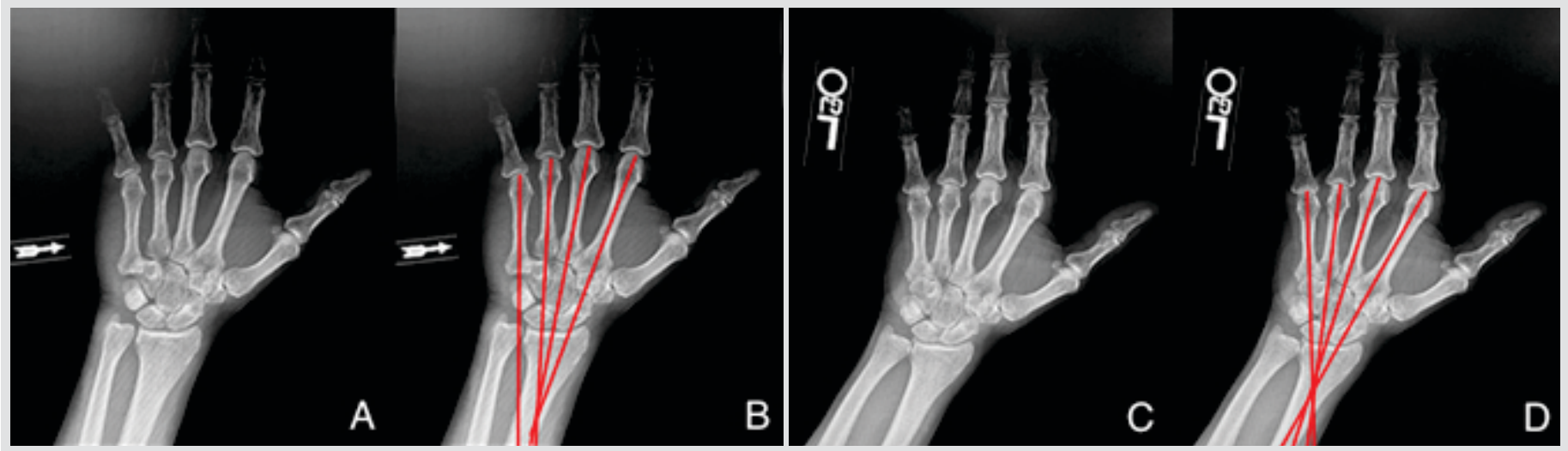

Figure 5: Metacarpal cascade lines. (a) Pre-operative posteroanterior view; (b) pre-operative posteroanterior view with metacarpal cascade lines; (c) post-operative posteroanterior view; (d) postoperative posteroanterior view with metacarpal cascade lines. Lines are drawn down the metacarpal shafts and should converge approximately $2 \mathrm{~cm}$ proximal to the distal radius articular surface. Preoperative radiographs demonstrate a clear divergence of the fifth metacarpal cascade line. 
longitudinal traction to the involved phalanx with volar or dorsal pressure applied over the base of the dislocated metacarpal to reverse the direction of dislocation. If the reduction can be held, an ulnar gutter splint or cast is applied and molded in an intrinsic plus position. Splinting is typically continued for 6 weeks with early repeat radiographs taken to ensure maintenance of reduction $[3,7,8]$. Displacement after closed reduction is typically seen within a 2 -week window; however, late displacement has been noted as late as 4 months $[11,12,13]$.

\section{Conclusion}

There are very limited data regarding closed reduction of chronic metacarpal dislocations. In our experience, an open reduction with excision of fibrous tissue was necessary to obtain an anatomic alignment of the metacarpal. Further, Kirschner wire fixation was required to maintain the reduction of the CMC joint. These pins were maintained for 6 weeks and resulted in no significant loss of digital range of motion or grip strength.

This paper provides a literature review on the diagnostic techniques and management options for the fifth CMC dislocations. It further provides a treatment algorithm for open reduction and Kirschner wire fixation of a chronic volar-ulnar fifth CMC dislocation resulting in an excellent outcome. A high index of suspicion for this disease entity should be present when evaluating patients with hand pain or subtle deformity with seemingly or reportedly negative radiographs.

\section{Clinical Message}

Isolated carpometacarpal dislocations are rare injuries that are frequently missed in the emergency department and urgent care settings. This paper presents a case of a chronic fifth carpometacarpal dislocation treated with open reduction and Kirschner wire fixation to review methods of diagnosing and treating these injuries.

\section{References}

1. Fisher M, Rogers L, Hendrix R. A systematic approach to the diagnosis of carpometacarpal dislocations. Radiographics 1982;2:612-27.

2. Fisher M, Rogers L, Hendrix R. Systematic approach to identifying fourth and fifth carpometacarpal joint dislocations. AJRAm J Roentgenol 1983;140:319-24.

3. McDonald L, Schupe P, Hammel N, Kroonen L. The intermetacarpal angle screening test for ulnar-sided carpometacarpal fracture-dislocations. J Hand Surg Am 2012;37:1839-44.

4. Wright A, Muir L. A review of published radiographic indicators of carpometacarpal dislocation including their application to volar dislocations through a case study. J Emerg Med 2015;49:e69-71.

5. Watanabe H, Osada R, Zukawa M, Kimura T. Isolated slipping dislocation in the ulnar direction of the fifth carpometacarpal joint-the utility of computed tomography. Inj Extra 2008;39:376-9.

6. Hodgson PD, Shewring DJ. The metacarpal cascade lines; use in the diagnosis of dislocations of the carpometacarpal joints.J Hand Surg Eur Vol 2007;32:277-81.

7. Storken G, Bogie R, Jansen E. Acute ulnar carpometacarpal dislocations. Can it be treated conservatively? A review of four cases. Hand (NY) 2011;6:420-3.

8. Berg E, Murphy D. Ulnopalmar dislocation of the fifth carpometacarpal joint-successful closed reduction: Review of the literature and anatomic reevaluation.J Hand Surg Am 1986;11:521-5.

9. Nalebuff E. Isolated anterior carpometacarpal dislocation of the fifth finger: Classification and case report. J Trauma 1968;8:1119-23.

10. Petrie R, Lamb D. Fracture-subluxation of base of fifth metacarpal. Hand 1974;6:82-6.

11. Garcia-Elias M, Bishop AT, Dobyns JH, Cooney WP, Linscheid RL. Transcarpal carpometacarpal dislocations, excluding the thumb.J Hand Surg Am 1990;15:531-40.

12. Hartwig R, Louis D. Multiple carpometacarpal dislocations. A review of four cases. J Bone Joint Surg Am 1976;61:906- 
8.

13. Hsu J, Curtis R. Carpometacarpal dislocations on the ulnar side of the hand.J Bone Joint Surg Am 1970;52:927-30.

\begin{tabular}{l} 
Conflict of Interest: Nil \\
Source of Support: Nil \\
\hline Consent: The authors confirm that informed consent was obtained \\
from the patient for publication of this case report
\end{tabular}

\section{How to Cite this Article}

Boltuch AD, Harker JN. Operative Intervention for a Chronic Fifth Carpometacarpal Dislocation: A Case Report and Review of Literature. Journal of Orthopaedic Case Reports 2021 January;11(1): 108-112. 